PROCEEDINGS OF THE

AMERICAN MATHEMATICAL SOCIETY

Volume 132, Number 7, Pages 2103-2113

S 0002-9939(04)07303-4

Article electronically published on January 27, 2004

\title{
THE IDEAL ENVELOPE OF AN OPERATOR ALGEBRA
}

\author{
DAVID P. BLECHER AND MASAYOSHI KANEDA
}

(Communicated by David R. Larson)

\begin{abstract}
A left ideal of any $C^{*}$-algebra is an example of an operator algebra with a right contractive approximate identity (r.c.a.i.). Conversely, we show here that operator algebras with a r.c.a.i. should be studied in terms of a certain left ideal of a $C^{*}$-algebra. We study operator algebras and their multiplier algebras from the perspective of "Hamana theory" and using the multiplier algebras introduced by the first author.
\end{abstract}

\section{INTRODUCTION AND NOTATION}

An operator algebra for us will mean a closed subalgebra of a $C^{*}$-algebra. A left ideal of any $C^{*}$-algebra is an example of an operator algebra with a right contractive approximate identity. Conversely, we study operator algebras with a right contractive approximate identity in terms of a certain left ideal of a $C^{*}$ algebra. We shall abbreviate "right (resp. left) contractive approximate identity" to "r.c.a.i." (resp. "l.c.a.i.").

In $\S 2$ of our paper we show that associated to any operator algebra $A$ with a r.c.a.i. there is an important left ideal $\mathfrak{J}_{e}(A)$ of a $C^{*}$-algebra $\mathcal{E}(A)$. We call $\mathfrak{J}_{e}(A)$ the "left ideal envelope" of $A$, and it may be used (see, e.g., the companion paper [3]) to deduce many general results about operator algebras with a r.c.a.i. from results about left ideals in a $C^{*}$-algebra. This is analogous to the case of operator algebras with a two-sided c.a.i., which are largely studied these days in terms of a certain $C^{*}$-algebra, namely the $C^{*}$-envelope. In $\S 3$ we study the "left multiplier operator algebra" of an operator algebra $A$ with a r.c.a.i. (which will be a theory symmetric to that of $R M(A)$ for an operator algebra $A$ with a l.c.a.i). This theory does not work out quite as nicely as the case when $A$ has a two-sided c.a.i., unless $A$ is a left ideal of a $C^{*}$-algebra.

We now list notation and background results which will be useful in various places. We reserve the letters $H, K$ for Hilbert spaces, and $J$ for a left ideal of a $C^{*}$-algebra. If $A$ is an algebra, then we write $\lambda: A \rightarrow \operatorname{Lin}(A)$ for the canonical "left regular representation" of $A$ on itself. By a "representation" $\pi: A \rightarrow B(H)$ of an operator algebra $A$ we shall mean a completely contractive homomorphism. If $A$ has a r.c.a.i. and if we say that $\pi$ is nondegenerate, then at the very least we

Received by the editors November 5, 2001 and, in revised form, April 16, 2003.

2000 Mathematics Subject Classification. Primary 46L05, 46L07, 47L30; Secondary 46H10, $47 \mathrm{~L} 75$.

Key words and phrases. Operator algebra, nonselfadjoint algebra, ideals.

This research was supported by a grant from the National Science Foundation. 
mean that $\pi(A) H$ is dense in $H$. Note that this last condition does not imply in general that $\pi\left(e_{\alpha}\right) \zeta \rightarrow \zeta$ for $\zeta \in H$ (where $\left\{e_{\alpha}\right\}$ is the r.c.a.i.), that one is used to in the two-sided case.

Lemma 1.1. Let $A$ be an operator algebra with either a l.c.a.i. or a r.c.a.i.. Suppose that $\pi: A \rightarrow B(H)$ is a completely contractive representation, let $K$ be the norm closure of $\pi(A) H$, and let $\pi^{\prime}: A \rightarrow B(K)$ be the induced representation. Then $\pi^{\prime}$ is a completely contractive homomorphism, and it is nondegenerate in the sense that $\pi^{\prime}(A) K$ is dense in $K$. Moreover, if $A$ has a r.c.a.i. and $\pi$ is isometric (resp. completely isometric, 1-1), then $\pi^{\prime}$ is also isometric (resp. completely isometric, $1-1)$.

Consequently, if $A$ is a subalgebra of $B(H)$ with a r.c.a.i., then we may view $A$ as a nondegenerate subalgebra of $B(K)$, where $K$ is the closure of $A H$.

Proof. That $\pi^{\prime}$ is a completely contractive homomorphism is clear. Clearly $\pi^{\prime}(A) K$ $\subset K$; however, if $a \in A$ and $\zeta \in H$, then $\pi(a) \zeta=\lim \pi^{\prime}(a) \pi\left(e_{\alpha}\right) \zeta \in \pi^{\prime}(A) K$ if $A$ has a r.c.a.i. Taking linear combinations of such expressions $\pi(a) \zeta$, and limits, shows that any element of $K$ is in the closure of $\pi^{\prime}(A) K$. So $\pi^{\prime}$ is nondegenerate in this case. A similar argument holds if $A$ has a l.c.a.i.

To see the statement here about the isometry, note that for $a \in A$ we have

$$
\begin{aligned}
\|a\|=\|\pi(a)\| & =\sup \{\|\pi(a) \zeta\|: \zeta \in \operatorname{Ball}(H)\} \\
& =\sup \lim _{\alpha}\left\{\left\|\pi^{\prime}(a) \pi\left(e_{\alpha}\right) \zeta\right\|: \zeta \in \operatorname{Ball}(H)\right\} .
\end{aligned}
$$

However, the right-hand side is dominated by $\left\|\pi^{\prime}(a)\right\|$, so that $\pi^{\prime}$ is an isometry. A similar argument works for a complete isometry. The 1-1 assertion is easier.

We will use without comment several very basic facts from $C^{*}$-algebra theory (see, e.g., [1] ), such as the basic definitions of the left multiplier algebra $L M(A)$ and the multiplier algebra $M(A)$ of a $C^{*}$-algebra. As a general reference for operator spaces the reader might consult [6, 10, 12]. We write ^ $: X \rightarrow X^{* *}$ for the canonical map; this is a complete isometry if $X$ is an operator space, and is a homomorphism if $X$ is an operator algebra, giving the second dual the Arens product [5].

We will often consider the basic examples $C_{n}$ (resp. $R_{n}$ ) of operator algebras with a right (resp. left) identity of norm 1; namely, the $n \times n$ matrices "supported on" the first column (resp. row). This is a left (resp. right) ideal of $M_{n}$, and has the projection $E_{11}$ as the one-sided identity.

If $X$ and $Y$ are subsets of an operator algebra, we usually write $X Y$ for the norm closure of the set of finite sums of products $x y$ of a term in $X$ and a term in $Y$. For example, if $J$ is a left ideal of a $C^{*}$-algebra $A$, then with this convention $J^{*} J$ will be a norm closed $C^{*}$-algebra. This convention extends to three sets; thus $J J^{*} J=J$ for a left ideal in a $C^{*}$-algebra, as is well known. These facts follow easily from the well-known results that a (norm closed) left ideal $J$ in a $C^{*}$-algebra has a (positive) right (contractive) approximate identity. Also $J \cap J^{*}=J^{*} J \subset J \subset J J^{*}$, so that $J$ is a left ideal of $J J^{*}$ (see [8], Lemma 4.1).

For the purposes of this paper we will define a triple system to be a norm closed subspace $X$ of a $C^{*}$-algebra such that $X X^{*} X \subset X$. It is well known that triple systems are "the same thing" as Hilbert $C^{*}$-modules, although there is a slight difference of emphasis in the two theories; the important structure on a triple system is the "triple product" $x y^{*} z$. A linear map $T: X \rightarrow Y$ between triple systems is a triple morphism if $T\left(x y^{*} z\right)=T(x) T(y)^{*} T(z)$ for all $x, y, z \in X$. Triple 
systems are operator spaces, and triple morphisms are completely contractive, and indeed are completely isometric if they are 1-1 (see [7]; this is related to results of Harris and Kaup). A completely isometric surjection between triple systems is a triple morphism. This last result might date back to around 1986, to Hamana, Kirchberg, and Ruan's Ph.D. thesis independently. See 7] or [2], A.5, for a proof.

Next we recall the left multiplier algebra $\mathcal{M}_{\ell}(X)$ of an operator space $X$. This is an operator algebra, which may be viewed as a subalgebra of $C B(X)$ containing $I d_{X}$, but with a different (bigger, in general) norm. Here $C B(X)$ is the space of completely bounded linear maps on $X$. Our first definition of $\mathcal{M}_{\ell}(X)$ (see [2]) was in terms of the "triple envelope" (defined below). For no very good reason, we will instead use a later equivalent formulation explained in Section 2 below. We will make much use of the following two simple results from [3]:

Lemma 1.2. Let $A$ be an operator algebra with a r.c.a.i. Then the canonical "left regular representation" $\lambda$ of $A$ on itself yields completely contractive embeddings (i.e. 1-1 homomorphisms)

$$
A \hookrightarrow \mathcal{M}_{\ell}(A) \hookrightarrow C B(A),
$$

and the first of these embeddings, and their composition, are completely isometric.

We remark that the canonical embedding $\mathcal{M}_{\ell}(A) \hookrightarrow C B(A)$, where $A$ is an operator algebra with a r.c.a.i., is not in general completely isometric, or even isometric (see Example 3.5).

Lemma 1.3. Suppose that $a \in B(H)$ and that $\left\{e_{\alpha}\right\}$ is a net of contractions in $B(H)$ such that $a e_{\alpha} \rightarrow a$. Then $a e_{\alpha} e_{\alpha}^{*} \rightarrow a$, ae $e_{\alpha}^{*} e_{\alpha} \rightarrow a$, and $a e_{\alpha}^{*} \rightarrow a$.

\section{The IDEAL EnVELOPE}

We begin by sketching Hamana's construction of the injective and the triple envelope of operator spaces. See, e.g., [7] for more details and notation; or [10], or the introductions of [4] and [2]. Let $X \subset B(H)$ be an operator space, and consider the Paulsen operator system

$$
\mathcal{S}(X):=\left[\begin{array}{cc}
\mathbb{C} 1_{H} & X \\
X^{*} & \mathbb{C} 1_{H}
\end{array}\right] \subset M_{2}(B(H)) .
$$

One then takes a minimal (with respect to a certain ordering) completely positive $\mathcal{S}(X)$-projection $\Phi$ on $M_{2}(B(H))$. By a well-known result of Choi and Effros, Im $\Phi$ is a $C^{*}$-algebra with the multiplication $\odot$ defined by $\xi \odot \eta:=\Phi(\xi \eta)$ for $\xi, \eta \in \operatorname{Im} \Phi$, and the other algebraic operations and norm are the usual ones. One may write

$$
\operatorname{Im} \Phi=\left[\begin{array}{cc}
I_{11} & I(X) \\
I(X)^{*} & I_{22}
\end{array}\right] \subset M_{2}(B(H)),
$$

where $I(X)$ is an injective envelope of $X$, and $I_{11}, I_{22}$ are injective $C^{*}$-algebras.

We continue to think of $I_{11}, I_{22}, I(X)$ and $I(X)^{*}$ as subsets of $B(H)$; however, the operation $\odot$ induces new products between elements of $I_{11}, I_{22}, I(X)$ and $I(X)^{*}$. To distinguish these multiplications from the original product on $M_{2}(B(H))$ we write the new products as $\circ$.

By a well-known trick one may also decompose

$$
\Phi=\left[\begin{array}{cc}
\psi_{1} & \phi \\
\phi^{*} & \psi_{2}
\end{array}\right]
$$



by

Also, one may write the $C^{*}$-subalgebra of $\operatorname{Im} \Phi$ (with the new product) generated

as

$$
\left[\begin{array}{cc}
0 & X \\
0 & 0
\end{array}\right]
$$

$$
\left[\begin{array}{cc}
\mathcal{E}(X) & \mathcal{T}(X) \\
\mathcal{T}(X)^{*} & \mathcal{F}(X)
\end{array}\right] \subset\left[\begin{array}{cc}
I_{11} & I(X) \\
I(X)^{*} & I_{22}
\end{array}\right]
$$

This defines $\mathcal{T}(X)$; it is clearly a $C^{*}$-module or triple system (viewed as the 1-2corner of the $C^{*}$-subalgebra just introduced, its triple product is $x \circ y^{*} \circ z$ ). Indeed, the span of expressions of the form $a_{1} \circ a_{2}^{*} \circ a_{3} \circ a_{4}^{*} \circ \cdots \circ a_{2 n+1}$, for $a_{i} \in X$, is dense in $\mathcal{T}(X)$. Thinking of $\mathcal{T}(X)$ as a triple system, and letting $i$ be the canonical map $X \rightarrow \mathcal{T}(X)$, we say that $(\mathcal{T}(X), i)$ is a triple envelope of $X$.

Lemma 2.1. Let $A$ be a subalgebra of $B(H)$, and suppose that $A$ has a r.c.a.i. $\left\{e_{\alpha}\right\}$. Then for all $a, b \in A$ we have (taking $X=A$ and using the notation above)

$$
a b=\lim _{\alpha} a \circ e_{\alpha}^{*} \circ b
$$

and

$$
\psi_{1}(a)=\lim _{\alpha} a \circ e_{\alpha}^{*} \in I_{11} .
$$

Proof. Using the notation above, and Lemma 1.3 we have

$$
\psi_{1}(a)=\lim _{\alpha} \psi_{1}\left(a e_{\alpha}^{*}\right)=\lim _{\alpha} a \circ e_{\alpha}^{*},
$$

the last step by definition of the o product. Similarly,

$$
a b=\phi(a b)=\phi\left(\lim _{\alpha} a e_{\alpha}^{*} b\right)=\lim _{\alpha} a \circ e_{\alpha}^{*} \circ b .
$$

We recall that an equivalent definition of $\mathcal{M}_{\ell}(X)$ given in [4] was as $I M_{\ell}(X)=$ $\left\{y \in I_{11}: y \circ X \subset X\right\}$. Using the last lemma, we see that if $A$ is an operator algebra with a r.c.a.i., then $\psi_{1}(a) \in I M_{\ell}(A)$ for all $a \in A$. Thus if $j$ is $\psi_{1}$ restricted to $A$, then $j: A \rightarrow I M_{\ell}(A)$. By the last lemma, $j(a) \circ b=a b$ for all $a, b \in A$, so that $j$ corresponds to the left regular representation $\lambda: A \rightarrow \mathcal{M}_{\ell}(A) \subset C B(A)$. We now show that $j$ is a completely isometric homomorphism. Indeed, if $x \in A$, then

$$
\|j(x)\|=\lim _{\alpha}\left\|x \circ e_{\alpha}^{*}\right\| \geq \lim _{\alpha}\left\|x \circ e_{\alpha}^{*} \circ e_{\beta}\right\|=\left\|x e_{\beta}\right\|
$$

using 2.1. Taking the limit shows that $j$ is isometric on $A$. A similar argument shows that $j$ is completely isometric. If $a, b \in A$, then

$$
j(a b)=\lim _{\alpha}(a b) \circ e_{\alpha}^{*}=\lim _{\alpha}\left(\lim _{\beta} a \circ e_{\beta}^{*} \circ b\right) \circ e_{\alpha}^{*}=\lim _{\alpha} j(a) \circ b \circ e_{\alpha}^{*}=j(a) \circ j(b) .
$$

Proposition 2.2. Let $A$ be an operator algebra with a r.c.a.i..

(1) Under the same assumptions as in Lemma 2.1, and notation as above,

$$
I M_{\ell}(A)=\left\{T \in I_{11}: T \circ j(A) \subset j(A)\right\} .
$$

(2) Elements of $\mathcal{M}_{\ell}(A)$, considered as maps on $A$, are right $A$-module maps. That is, $\mathcal{M}_{\ell}(A) \subset C B_{A}(A)$ as sets. Also, $\mathcal{M}_{r}(A) \subset{ }_{A} C B(A)$ as sets. 
Proof. (1) Let $a \in A$; then $T \circ j(a)=\lim _{\alpha} T \circ a \circ e_{\alpha}^{*}$, which equals $j(T \circ a)$ if $T \in I M_{\ell}(A)$. Conversely, if $T \circ j(a)=j\left(a^{\prime}\right)$ for an $a^{\prime} \in A$, then by the last formula and 2.1 we have $T \circ a e_{\beta}=T \circ j(a) \circ e_{\beta}=a^{\prime} e_{\beta}$, so that $T \circ a=a^{\prime} \in A$.

(2) For the last assertion, if $T \in \mathcal{M}_{r}(A)$, then there exists $y \in I_{22}$ such that $A \circ y \subset A$, and $T$ is the map $T(a)=a \circ y$. Hence, for $c \in A$,

$$
T(c a)=(c a) \circ y=\lim _{\alpha} c \circ e_{\alpha}^{*} \circ a \circ y=c T(a),
$$

using 2.1 twice. The other is similar.

Theorem 2.3. Let $A$ be an operator algebra with a r.c.a.i., let $(I(A), i)$ be its injective envelope discussed above 2.1, and let $B$ be the injective unital $C^{*}$-algebra $I_{11}$ (discussed above). Then there is an orthogonal projection $e \in B$ such that $I^{\prime}(A)=(B \circ e, j)$ is also an injective envelope for $A$, where $j$ is as above. The completely isometric embedding $j: A \rightarrow I^{\prime}(A) \subset B$ is a homomorphism. Moreover, if $A$ has a right identity $f$ of norm 1 , then one may take $e=j(f)$.

Before we prove this, we remark that we may define the ideal injective envelope of such an operator algebra $A$ to be $I^{\prime}(A)$ as in the theorem.

Proof. Define $\psi: I_{11} \circ A \rightarrow I_{11}$ by $\psi(x)=\lim _{\alpha} x \circ e_{\alpha}^{*}$. By 2.1 this limit exists, and $\psi$ is clearly completely contractive. Note that $\psi$ is also a left $I_{11}$-module map, so that by a result in [4] we may extend $\psi$ to a completely contractive left $I_{11}$-module map $\hat{\psi}: I(A) \rightarrow I_{11}$. The restriction of this map to $A$ was the map called $j$ above, which we showed above 2.2 was a completely isometric homomorphism. By the "essential property" of $I(A), \hat{\psi}$ is a complete isometry. By [4], 2.7, there is a $v \in \operatorname{Ball}(I(A))$ such that $\hat{\psi}(x)=x \circ v^{*}$, for all $x \in I(A)$. The range of $\hat{\psi}$ is a left ideal of $I_{11}$, so that $\hat{\psi}$ is a triple morphism by a fact stated towards the end of the introduction.

Next define a map $I_{11} \circ j(A) \subset I_{11} \rightarrow I(A)$ by $z \mapsto \lim _{\alpha} z \circ e_{\alpha}$. To see that this limit exists, note that for $x \in I_{11}$ and $a \in A$ we have, by the type of calculations found in the proof of 2.2, that $\lim _{\alpha} x \circ j(a) \circ e_{\alpha}=x \circ a$. So the limit exists on $I_{11} \circ j(A)$, and moreover this map is exactly the map $x \circ j(a) \mapsto x \circ a$. It is thus a completely contractive left $I_{11}$-module map, and extends by [4] to a completely contractive left $I_{11}$-module map $\mu: I_{11} \rightarrow I(A)$. There clearly exists $w \in \operatorname{Ball}(I(A))$ such that $\mu=-\circ w$. Now notice that the composition $\mu \hat{\psi}$ is a completely contractive map $I(A) \rightarrow I(A)$, which restricts to the identity map on $A$. By the "rigidity property" of $I(A)$ we have $\mu \hat{\psi}=I d$ on $I(A)$. Thus $\hat{\psi}$ is a complete isometry, and $\mu$ is onto. Moreover, since $x \circ\left(v^{*} \circ w\right)=x$ for all $x \in I(A)$, it follows from [4], Corollary 1.3 , that $v^{*} \circ w$ is the identity of $I_{22}$. Thus by a well-known operator theory fact, $v=w$. A similar argument shows that $v$ above is unique. We define $e=v \circ v^{*} \in I_{11}=B$; this is an orthogonal projection. Moreover, $\operatorname{Ran} \hat{\psi}=B \circ e$, as may be seen easily from the above. It follows immediately that $(B \circ e, j)$ is an injective envelope for $A$, which is a homomorphism. Finally, if $A$ has a right identity $f$ of norm 1 , then we may take $v$ above to be $f$, so that $j(f)=f \circ f=e$.

Corollary 2.4. Let $A$ be an operator algebra with a r.c.a.i. $\left\{e_{\alpha}\right\}$. Suppose that $(\mathcal{T}(A), i)$ is a triple envelope for $A$, and let $\mathcal{E}(A)=\mathcal{T}(A) \mathcal{T}(A)^{*}$. The map $\psi$ : $\mathcal{T}(A) \longrightarrow \mathcal{E}(A)$ defined by $\psi(x):=\lim _{\alpha} x i\left(e_{\alpha}\right)^{*}$ is a well-defined complete isometry, and $\psi \circ i$ is a homomorphism $j$ on $A$. The range of $\psi$ is a left ideal $J$ in the $C^{*}$ algebra $\mathcal{E}(A)$, and $\psi$ is also a triple morphism onto $J$. Thus $(J, j)$ is another 
triple envelope of $A$. Moreover, $\psi(x) \psi(y)^{*}=x y^{*}$ for any $x, y \in \mathcal{T}(A)$, and hence $J J^{*}=\mathcal{E}(A)$.

Proof. By "abstract nonsense" we can assume that $(\mathcal{T}(A), i)$ is the triple envelope considered above 2.1 (in short, because the statement we are attempting to prove is invariant under the notion we called Hilbert $A$-isomorphism in [2], Appendix A). Since $\mathcal{E}(A) \subset I_{11}$, and since $\mathcal{T}(A)=\mathcal{E}(A) \circ A$, the map $\psi$ in our statement is a restriction of the map of the same name in Theorem 2.3. All claims then follow from 2.3 For example, $\psi(x) \circ \psi(y)^{*}=x \circ v^{*} \circ v \circ y^{*}=x \circ y^{*}$, since we showed that $v^{*} \circ v$ is the identity of $I_{22}$.

This corollary also may be proved without 2.3. It is clear from 2.4 that for an operator algebra $A$ with a r.c.a.i., $\mathcal{T}(A)$ may be taken to be a left ideal in a $C^{*}$-algebra, and this left ideal therefore possesses Hamana's universal property of the triple envelope (see [7 or [2, Appendix A). As a special case, note that this observation immediately gives an abstract characterization of left ideals in $C^{*}$ algebras as exactly the operator algebras $A$ with a r.c.a.i. that are also "abstract triple systems" (note that for such $A$, we have $\mathcal{T}(A)=A$, by the universal property of the triple envelope). See [3] for some related facts.

We say that a pair $(J, i)$ consisting of a left ideal $J$ in a $C^{*}$-algebra, and a completely isometric homomorphism $i: A \rightarrow J$, is a left ideal extension of an operator algebra $A$ if $i(A)$ "generates $J$ as a triple system". That is, the span of expressions of the form $i\left(a_{1}\right) i\left(a_{2}\right)^{*} i\left(a_{3}\right) i\left(a_{4}\right)^{*} \cdots i\left(a_{2 n+1}\right)$, for $a_{i} \in A$, are dense in $J$. It follows from this that $\left\{i\left(e_{\alpha}\right)\right\}$ is a r.c.a.i. for $J$ if $\left\{e_{\alpha}\right\}$ is a r.c.a.i. for $A$. Then it is clear from 2.4 that $(\mathcal{T}(A), j)$ may be taken to be a left ideal extension of $A$, and it is the "minimal such". The new point in the corollary below is that $\tau$ may be chosen to be a homomorphism:

Corollary 2.5. Let $A$ be an operator algebra with a r.c.a.i.. Then there exists a left ideal extension $\left(\mathfrak{J}_{e}(A), j\right)$ of $A$, with $\mathfrak{J}_{e}(A)$ a left ideal in a $C^{*}$-algebra $\mathcal{E}(A)=$ $\mathfrak{J}_{e}(A) \mathfrak{J}_{e}(A)^{*}$, such that for any other left ideal extension $(J, i)$ of $A$, there exists a (necessarily unique and surjective) completely contractive homomorphism $\tau: J \rightarrow$ $\mathfrak{J}_{e}(A)$, which is also a triple morphism, such that $\tau \circ i=j$. Thus $J /(\operatorname{ker} \tau) \cong$ $\mathfrak{J}_{e}(A)$ completely isometrically homomorphically (i.e., as operator algebras and as triple systems). Moreover, $\left(\mathfrak{J}_{e}(A), j\right)$ is unique in the following sense: given any other $\left(J^{\prime}, j^{\prime}\right)$ with this universal property, then there exists a surjective completely isometric homomorphism $\theta: \mathfrak{J}_{e}(A) \rightarrow J^{\prime}$ such that $\theta \circ j=j^{\prime}$.

Proof. The uniqueness is fairly obvious and standard. The existence follows from 2.4 (setting $\mathfrak{J}_{e}(A)=(J, j)$ there), together with Hamana's universal property for the "triple envelope" mentioned above. Indeed, we have

$$
\tau(x y)=\lim \tau\left(x i\left(e_{\alpha}\right)^{*} y\right)=\lim \tau(x) j\left(e_{\alpha}\right)^{*} \tau(y)=\tau(x) \tau(y)
$$

for $x, y \in J$, using Lemma 1.3 and the observations above.

We call $\left(\mathfrak{J}_{e}(A), j\right)$ the left ideal envelope of $A$.

\section{The Left multiplier Algebra of AN AlgeBra With R.C.A.I.}

This theory is rather different from the case of the left multiplier algebra of an operator algebra with a l.c.a.i., which was treated in [3]. Part of this may be seen by considering the basic example of $A=C_{n}$ (the $n \times n$ matrices "supported on" 
the first column). It is fairly clear that its left multiplier operator algebra "should be" $M_{n}$.

After a little thought about operator algebras with a r.c.a.i., one comes up with the following list (which we shall refer to throughout this section) of "possible candidate definitions" for $\operatorname{LM}(A)$ in this case (all of which contain $A$ completely isometrically):

(I) $\left\{x \in A^{* *}: x \hat{A} \subset \hat{A}\right\}$,

(II) $\{T \in B(H): T \pi(A) \subset \pi(A)\}$, where $\pi: A \rightarrow B(H)$ is a completely isometric nondegenerate representation,

(III) $\mathcal{M}_{\ell}(A)$,

(IV) $C B_{A}(A)$ (right module maps),

(V) the "maximum essential left multiplier extension of $A$ ".

We will spell out later what is meant in this setting by "essential left multiplier extension". One can rule out (I) fairly quickly as a plausible candidate, since it is not unital (e.g., take $A=C_{n}$ ). However, one would hope that most of the other four items are completely isometrically isomorphic. Unfortunately, most of the equivalences amongst these items break down if $A$ is a general operator algebra with a r.c.a.i.. Nonetheless, one of these five candidates will emerge from our study below as the best candidate for $L M(A)$.

In the special case of a left ideal $J$ in a $C^{*}$-algebra, we will take the term "essential left multiplier extension", used in $(\mathrm{V})$, to mean a pair $(B, \pi)$ consisting of an operator algebra $B$ with identity of norm 1, together with a completely isometric homomorphism $\pi: A \rightarrow B$, such that $\pi(A)$ is a left ideal of $B$, and such that the natural map $B \rightarrow C B(A)$ is completely isometric. There is a natural ordering, and corresponding notion of equivalence, of "left multiplier extensions" of $A$, which we will not bother to spell out. Of particular interest, in (II), are the ideal representations of $J$ discussed in [3], that is, the completely isometric representations $\pi: J \rightarrow B(H)$ that are restrictions of faithful *-representations $J J^{*} \rightarrow B(H)$ (or, equivalently, the completely isometric representations of $J$ that are also triple morphisms).

Theorem 3.1. Let $J$ be a left ideal of a $C^{*}$-algebra. The algebras in (III)-(V) are all completely isometrically isomorphic to LM(JJ*). Any algebra in (II)-(V) contains the algebra in (I) completely isometrically isomorphically as a (proper, in general) subalgebra. If, further, in (II) we only consider 1-1 nondegenerate ideal representations $\pi$, then the algebras in (II)-(V) are all completely isometrically isomorphic. Also $B_{J}(J)=C B_{J}(J)$ isometrically.

Proof. In this case $J$ is a $C^{*}$-module, and $\mathcal{M}_{\ell}(J)$ may easily be seen, from [2, A.4, if necessary, to be $L M\left(J J^{*}\right)$. Clearly $C B_{J}(J) \subset C B_{J^{*} J}(J)$. Conversely, if $T \in C B_{J^{*} J}(J)$, then for $x, y \in J$ we have, using Lemma 1.3, that $T(x y)=$ $\lim T\left(x e_{\alpha}^{*} y\right)=\lim T(x) e_{\alpha}^{*} y=T(x) y$, if $\left\{e_{\alpha}\right\}$ is a r.c.a.i. in $J$. Thus $C B_{J}(J)=$ $C B_{J^{*} J}(J)$. By a theorem of Lin $\left([9,1.4) B_{J^{*} J}(J)=L M\left(J J^{*}\right)\right.$. The operator space version of this is true too (see [1]); that is, $C B_{J^{*} J}(J)=L M\left(J J^{*}\right)$ completely isometrically. Thus (III) $=(\mathrm{IV})$, and we obtain the last assertion of the statement of the theorem too. It is now clear that (IV) is an essential left multiplier extension, so that it is the biggest such. That is, (IV) $=(\mathrm{V})$.

We will defer until[3.2 the proof that an algebra in (II) contains the algebra in (I) completely isometrically. Finally, given a nondegenerate faithful *-representation 
$\theta: J J^{*} \rightarrow B(H)$, we know from the multiplier theory for $C^{*}$-algebras (see, e.g., [1], 3.1.12) that $L M\left(J J^{*}\right)=\left\{T \in B(H): T \theta\left(J J^{*}\right) \subset \theta\left(J J^{*}\right)\right\}$. However, since for any ideal $J$ we have $J=J J^{*} J$, if $T \in B(H)$, then $T \theta(J) \subset \theta(J)$ if and only if $T \theta\left(J J^{*}\right) \subset \theta\left(J J^{*}\right)$. Thus the algebra in (II) equals $L M\left(J J^{*}\right)$ too in this case.

We have now completed our discussion of $L M(J)$ for a left ideal $J$ in a $C^{*}$ algebra, and turn to the more general case of an arbitrary operator algebra $A$ with a r.c.a.i. We define a left multiplier extension of $A$ to be a pair $(B, \pi)$ consisting of an operator algebra $B$ with an identity of norm 1 , and a completely isometric homomorphism $\pi: A \rightarrow B$ such that $\pi(A)$ is a left ideal of $B$. We say that $(B, \pi)$ is an essential left multiplier extension of $A$ if in addition the canonical map $B \rightarrow B(A)$ is $1-1$. Note that this canonical map is then a completely contractive homomorphism into $C B_{A}(A)$ (viewing $A$ as a right $A$-module). In the two-sided case $C B_{A}(A)$ is itself a left multiplier extension of $A$, and is therefore the largest left multiplier extension, but this is not true in general for us (see example 3.4). We write $(B, \pi) \leq\left(B^{\prime}, \pi^{\prime}\right)$ if there exists a completely contractive homomorphism $\theta: B \rightarrow B^{\prime}$ such that $\theta \circ \pi=\pi^{\prime}$. We say that two left multiplier extensions $(B, \pi)$ and $\left(B^{\prime}, \pi^{\prime}\right)$ are $A$-equivalent if there exists a completely isometric isomorphism $\theta: B \rightarrow B^{\prime}$ with $\theta \circ \pi=\pi^{\prime}$. This is an equivalence relation, and " $\leq$ " induces a welldefined ordering on the equivalence classes. To see this, note that if $\theta: B \rightarrow B$ is a completely contractive homomorphism extending the identity mapping on $\pi(A)$, then $\theta$ is the identity mapping. Indeed, $\theta(b) \pi(a)=\theta(b \pi(a))=b \pi(a)$, so that $\theta(b)-b$ is in the kernel of the canonical map $B \rightarrow C B(A)$. It follows that if there exists a maximum essential left multiplier extension of $A$, then it is unique up to $A$-equivalence. We remark further that if two left multiplier extensions are $A$-equivalent, and if one is essential, then so is the other. We leave the proof of this as an easy exercise.

One more definition: we say that a representation $\pi: A \rightarrow B(H)$ is a completely isometric Shilov representation, if $\pi$ is the restriction of a 1-1*-homomorphism $\mathcal{E}(A) \rightarrow B(H)$ (viewing $A \subset \mathcal{E}(A)$ as in 2.5 say, via the completely isometric homomorphism $j$ there). Note that the "ideal representations" $\pi$ considered in Theorem 3.1 also happen to be Shilov representations.

Theorem 3.2. Let $A$ be an operator algebra with a r.c.a.i.. Then $\mathcal{M}_{\ell}(A)$ is the (unique up to A-equivalence) maximum essential left multiplier extension of $A$. That is, the algebras in items (III) and (V) in the list at the beginning of $\S 3$ are completely isometrically isomorphic. Also $\mathcal{M}_{\ell}(A)$ contains, and dominates in the ordering above, the essential left multiplier extensions described in (II), which in turn contain the algebra in (I) completely isometrically isomorphically. However, if $\pi$ in (II) is a Shilov representation of $A$, then the algebra in (II) is completely isometrically isomorphic to (III) and (V). The algebra in (IV), namely $C B_{A}(A)$, is not in general an operator algebra, and its subalgebra which corresponds to the algebra in (III) (and $(V)$ ) does not correspond isometrically. That is, the canonical 1-1 homomorphic embedding of $\mathcal{M}_{\ell}(A)$ into $C B_{A}(A)$ (or even $B_{A}(A)$ ) need not be isometric.

Proof. That the canonical inclusion of (III) in (IV) is not isometric may be seen in Example 3.5. That $C B_{A}(A)$ is not in general an operator algebra may be seen in Example 3.4 
We have seen in 2.2 that $\left(\mathcal{M}_{\ell}(A), \lambda\right)$ is an essential left multiplier extension. It is evident from the fundamental properties of $\mathcal{M}_{\ell}(A)$ (from Theorem 4.10 (1) of [2], say) that given any essential left multiplier extension $(B, \pi)$ of $A$, there is a canonical completely contractive homomorphism $\sigma: B \rightarrow \mathcal{M}_{\ell}(A)$. It is obvious that via this homomorphism $(B, \pi) \leq\left(\mathcal{M}_{\ell}(A), \lambda\right)$. So $\left(\mathcal{M}_{\ell}(A), \lambda\right)$ is the maximum essential left multiplier extension.

If $\pi: A \rightarrow B(H)$ is a completely isometric "nondegenerate representation" (by which we mean at least that $\pi(A) H$ is dense in $H)$, then $\{T \in B(H): T \pi(A) \subset$ $\pi(A)\}$ is fairly evidently an essential left multiplier extension of $A$, and hence is dominated by $\mathcal{M}_{\ell}(A)$ (by the fact in the previous paragraph).

We now show that any algebra in (II) contains (I) completely isometrically. If $\pi: A \rightarrow B(H)$ is any (not necessarily nondegenerate) completely isometric homomorphism, consider the following sequence of completely contractive homomorphisms:

$$
A \hookrightarrow A^{* *} \stackrel{\pi^{* *}}{\rightarrow} B(H)^{* *} \rightarrow B(H)
$$

where the first and last maps here are the canonical inclusion and projection. Let $\sigma$ be the composition of the last two maps, restricted to $\left\{x \in A^{* *}: x \hat{A} \subset \hat{A}\right\}$. Then $\sigma(\hat{a})=\pi(a)$ for $a \in A$, and so for $x$ as above and $a \in A$ we have

$$
\sigma(x) \pi(a)=\sigma(x \hat{a}) \in \sigma(\hat{A})=\pi(A) .
$$

Thus $\sigma$ maps into $\{T \in B(H): T \pi(A) \subset \pi(A)\}$. To see that $\sigma$ is isometric, note that

$$
\|\sigma(x)\| \geq\left\|\sigma(x) \pi\left(e_{\alpha}\right)\right\|=\left\|\sigma\left(x \hat{e}_{\alpha}\right)\right\|=\left\|\pi\left(x \hat{e}_{\alpha}\right)\right\|=\left\|x \hat{e}_{\alpha}\right\| .
$$

Now the right identity of norm 1 of $A^{* *}$ is a weak* limit point of the $\left\{\hat{e}_{\alpha}\right\}$, and using the separate weak* continuity of the product on $A^{* *}$, we see that $\|\sigma(x)\| \geq\|x\|$. Similarly, $\sigma$ is completely isometric.

Finally, we discuss the equivalence of (III) with (II) for Shilov representations $\pi$. Take $\pi: \mathcal{E}(A) \rightarrow B(H)$ to be any nondegenerate $1-1 *$-homomorphism. Then it is easy to see that $\pi_{\left.\right|_{A}}$ is a nondegenerate completely isometric homomorphism. By the basic theory of multipliers of $C^{*}$-algebras we may view $L M(\mathcal{E}(A))=\{T \in$ $B(H): T \pi(\mathcal{E}(A)) \subset \pi(\mathcal{E}(A))\}$. From the remark after Proposition 2.2 we thus have $\mathcal{M}_{\ell}(A)$ completely isometrically isomorphic to

$$
\begin{gathered}
\{T \in B(H): T \pi(\mathcal{E}(A)) \subset \pi(\mathcal{E}(A)) \text { and } T \pi(A) \subset \pi(A)\} \\
=\{T \in B(H): T \pi(A) \subset \pi(A)\}
\end{gathered}
$$

since $j(A) j(A)^{*}$ generates $\mathcal{E}(A)$ as a $C^{*}$-algebra.

Proposition 3.3. Let $A$ be an operator algebra with a r.c.a.i. Then $C B_{A}(A)$ is an operator algebra if and only if $C B_{A}(A)$ is completely isometrically isomorphic to $\mathcal{M}_{\ell}(A)$.

Proof. The one direction is trivial since $\mathcal{M}_{\ell}(A)$ is an operator algebra. To see the other, note that by 1.2 and 2.2 we have $\lambda(A) \subset C B_{A}(A)$ and $\mathcal{M}_{\ell}(A) \subset C B_{A}(A)$ (although the $\mathcal{M}_{\ell}(A)$-norm is possibly larger than the $C B_{A}(A)$-norm). Thus if $C B_{A}(A)$ is an operator algebra, then $\left(C B_{A}(A), \lambda\right)$ is an essential left multiplier extension of $A$. Thus there exists a 1-1 completely contractive homomorphism $C B_{A}(A) \rightarrow \mathcal{M}_{\ell}(A)$ (by the equivalence of (III) and (V) in [3.2). It is easy to see that this homomorphism restricts to the identity map on $\mathcal{M}_{\ell}(A)$, whose inverse is completely contractive as a map $\mathcal{M}_{\ell}(A) \rightarrow C B_{A}(A)$. 
Next we consider some examples:

Example 3.4. Let $A \subset M_{3}$ be the subalgebra supported on the 1-2, 1-3, 2-2, and 3-3 entries only:

$$
A=\left[\begin{array}{lll}
0 & * & * \\
0 & * & 0 \\
0 & 0 & *
\end{array}\right] .
$$

Then $A$ is an operator algebra with a right identity of norm 1 . One may easily compute $\mathfrak{J}_{e}(A)=\mathcal{T}(A)=I(A)=M_{3,2}$, and $\mathcal{E}(A)=M_{3}$, from which it follows that $\mathcal{M}_{\ell}(A)$ is the subalgebra of $M_{3}$ spanned by $A$ and $I_{3}$. Thus $\mathcal{M}_{\ell}(A)$ is 5 -dimensional. On the other hand, the algebra given by item (I) in the list at the beginning of $\S 3$ is $A$ again, which is 4-dimensional. A tedious but completely elementary algebraic computation shows that $C B_{A}(A)$, item (IV) on the aforementioned list, is 8-dimensional. This shows by 3.3 that $C B_{A}(A)$ cannot be completely isometrically isomorphic to an operator algebra (in contrast to the two-sided c.a.i. case).

Example 3.5. It is perhaps true in the previous example that $\mathcal{M}_{\ell}(A)$ is not contained isometrically in $C B(A)$, but this seems difficult to check. Instead we generalize this example to an interesting example of an operator algebra $A$ with a right identity of norm 1 , for which $\mathcal{M}_{\ell}(A)$ is fairly clearly not contained isometrically in $C B(A)$ (or $B(A)$ ). To do this we take a unital $C^{*}$-algebra $B$ with no nontrivial two-sided ideals, and a closed subspace $X \subset B$ that generates $B$ as a triple system. To be more concrete, one could take $B=M_{3}$ and $X \subset M_{3}$ as in Example 4.4 of [2]. Consider $A \subset M_{3}(B)$, the subalgebra supported on the 1-2, 1-3, 2-2, and 3-3 entries only (i.e., zero elsewhere), which has scalar multiples of the identity operator in the 2-2 and 3-3 entries, and has operators from $X$ in the 1-2 and 1-3 entries. The triple system generated by $A$ in $M_{3}(B)$ is $M_{3,2}(B)$, similarly to the previous example. As in that example, one sees that $\mathcal{E}(A)=M_{3}(B)$ and $\mathcal{M}_{\ell}(A)$ is the subalgebra of $M_{3}(B)$ spanned by $A$ and matrices supported only in the 1-1 entry, and that entry may be anything in $\mathcal{M}_{\ell}(X)$. Now consider one of these last matrices, $T$ say, and let $a$ be its one nonzero entry (in $\mathcal{M}_{\ell}(X)$ ). Viewing $T \in C B(A)$ as a left multiplication map, it is clear that its norm or "cb-norm" is the same as the norm or "cb-norm" of its 1-1 entry, as a left multiplication map on $X$. But in Example 4.4 of [2] it is shown that neither of these numbers equals $\|a\|=\|T\|$ in general.

\section{ACKNOWLEDGMENT}

Several results in this paper were included as part of the second author's Ph.D. thesis, done under the direction of the first author during the 2000-2001 academic year. He expresses his gratitude to the first author for his kind instruction and advising.

\section{REFERENCES}

[1] D. P. Blecher, A new approach to Hilbert $C^{*}{ }_{-}$modules, Mathematische Annalen 307 (1997), 253-290. MR 98d:46063

[2] D. P. Blecher, The Shilov boundary of an operator space, and the characterization theorems, Journal of Functional Analysis 182 (2001), 280-343. MR 2002d:46049

[3] D. P. Blecher, One-sided ideals and approximate identities in operator algebras, to appear in Journal of the Australian Mathematical Society.

[4] D. P. Blecher and V. I. Paulsen, Multipliers of operator spaces, and the injective envelope, Pacific Journal of Mathematics 200 (2001), 1-17. MR 2002k:46150 
[5] F. F. Bonsall and J. Duncan, Complete normed algebras, Springer-Verlag, New YorkHeidelberg (1973). MR 54:11013

[6] E. G. Effros and Z. J. Ruan, Operator Spaces, The Clarendon Press, Oxford University Press, New York, 2000. MR 2002a:46082

[7] M. Hamana, Triple envelopes and Šilov boundaries of operator spaces, Math. J. Toyama University 22 (1999), 77-93. MR 2001a:46057

[8] M. Kaneda and V. I. Paulsen, Characterizations of essential ideals as operator modules over $C^{*}$-algebras, Journal of Operator Theory 49 (2003), 245-262.

[9] H. Lin, Bounded module maps and pure completely positive maps, J. Operator Theory 26 (1991), 121-138. MR 94f:46071

[10] V. I. Paulsen, Completely bounded maps and operator algebras, Cambridge Univ. Press, 2002.

[11] G. Pedersen, C*-algebras and their automorphism groups, Academic Press, 1979. MR 81e:46037

[12] G. Pisier, Introduction to operator space theory, London Mathematical Society Lecture Note Series, no. 294, Cambridge University Press, Cambridge, 2003.

[13] Y.-T. Poon and Z.-J. Ruan, Operator algebras with contractive approximate identities, Canadian Journal of Mathematics 46 (1994), 397-414. MR 95d:47057

[14] Z.-J. Ruan, Injectivity of operator spaces, Trans. Amer. Math. Soc. 315 (1989), 89-104. MR 91d:46078

Department of Mathematics, University of Houston, 4800 Calhoun Road, Houston, TEXAS 77204-3008

E-mail address: dblecher@math.uh.edu

URL: http://www. math.uh.edu/ dblecher/

Department of Mathematics, University of California, Irvine, California 92697-3875

E-mail address: mkaneda@math.uci.edu

URL: http://www. math.uci.edu/ mkaneda 